\title{
Un ping-pong transatlantique: les traductions de Proust en espagnol
}

\author{
Thomas BARÈGE \\ Université de Valenciennes-France
}

El estudio que sigue presenta las distintas traducciones en español, sean españolas o argentinas, de la obra maestra de Marcel Proust, $\grave{A}$ la recherche du temps perdu; comenta la historia de esas traducciones y demuestra que los factores exteriores influyen sobre el destino de cada traducción. Además hacemos una comparación de las calidades de cada traducción intentando determinar cuál es la mejor.

PAlabRas ClaVE: traducción, Proust, español, Argentina.

This article is about Spanish translations of $\dot{A}$ la recherche du temps perdu's Marcel Proust. We study all the translations in an historical perspective and analyze how external issues influence the translation's reception. Finally, we try to estimate which one is the best.

KEY WORDS: translation, Proust, spanish, Argentina.

2013 est l'occasion de célébrer le centenaire de la publication de Du côté de chez Swann, premier volume de $\grave{A}$ la recherche du temps perdu, la grande —et presque unique- œuvre de Proust. C'est sans doute le moment idéal pour rappeler que le monde hispanique fut le premier à l'étranger à reconnaître par le moyen de la traduction l'importance de cette œuvre, et ce, dès 1920. L'histoire des traductions de Proust en espagnol est loin d'être simple et linéaire, à vrai dire, elle s'apparente à un roman avec ses rebondissements mêlant les anecdotes plus personnelles à la grande Histoire. ${ }^{1}$

Proust a été traduit et retraduit à diverses reprises malgré le fait qu'il s'agisse d'un auteur du $\mathrm{XX}^{\mathrm{e}}$ siècle, ce qui laisse donc moins de latitude pour les retraductions. Les lecteurs hispanophones bénéficient de six traductions complètes, espagnoles et argentines, ce qui peut surprendre pour une œuvre extrêmement volumineuse comme l'est

\footnotetext{
${ }^{1}$ Je rappelle, pour les lecteurs qui ne sont guère familiers de l'œuvre de Proust, la date de publication de chaque volume de la Recherche: Du côté de chez Swann (1913), À l'ombre des jeunes filles en fleurs (1919), Le côté de Guermantes (1921), Sodome et Gomorrhe (1922) La prisonnière (1922), Albertine disparue (1925) Le temps retrouvé (1927).

Le lecteur trouvera une bibliographie qui référence toutes les éditions hispaniques à la fin de cette étude.
} 
la Recherche. C'est en soi un cas assez rare de trouver autant de retraductions pour une œuvre du XX $\mathrm{X}^{\mathrm{e}}$ siècle comptant plusieurs milliers de pages.

Lorsque l'on traduit à nouveau un texte, on traduit toujours en tenant compte de la ou des traductions antérieures. Il s'agit bel et bien d'une retraduction. Il s'établit alors un lien avec les traductions antérieures, un lien de concurrence, la nouvelle traduction corrigeant toujours dans une certaine mesure — même sans le dire explicitement — la traduction précédente. Il se crée ainsi un va et vient entre les traductions qui est parfois plein de tensions, et le cas de Proust est particulièrement riche à cet égard.

Les discours qui ont accompagné la publication des différentes traductions ont pour certains été très durs envers le travail réalisé. Cette dureté s'explique par des raisons liées à la qualité des traductions, mais le fait que ces traductions soient ou espagnoles, ou argentines, n'est pas anodin: on peut voir à l'œuvre dans cette opposition, presque une vieille rivalité post-coloniale. On n'évoquera pas ici les retraductions partielles — qui sont assez nombreuses elles aussi- qu'il s'agisse de courts passages ou bien des parties entières de la Recherche, c'est le cas notamment pour Un amour de Swann, retraduit à plusieurs reprises. Pour les seules années 2000, trois traductions complètes ont été lancées, ce qui peut étonner et qui montre aussi l'intérêt que le monde hispanique porte à Proust.

\section{Le corpus}

Tout commence avec une histoire personnelle, celle d'un assistant d'espagnol à la Sorbonne qui découvre Proust et se met à le traduire à son retour en Espagne: il s'agit de Pedro Salinas qui deviendra le poète que l'on sait. ${ }^{2}$ Ainsi en 1920 est publié, seulement sept ans après la publication française, Por el camino de Swann chez Espasa-Calpe à Madrid. Deux ans plus tard, c'est le second tome de la Recherche qui paraît sous le titre $A$ la sombra de las muchachas en flor, toujours avec le même éditeur et le même traducteur.

Tout débute donc sous les meilleurs auspices, avec une traduction rapide, par un traducteur qui deviendra très prestigieux et chez un grand éditeur. Il s'agit alors de la première traduction de Proust dans le monde. Pour des raisons qui sont plus ou moins bien connues, l'enthousiasme des débuts disparaît progressivement chez Salinas. Le troisième tome ne sera publié qu'en 1931 et 1932 (en deux volumes, comme dans l'édition française) avec un certain retard, il faudra même avoir recours à un autre traducteur (José María Quiroga Plá) pour suppléer Salinas qui a semble-t-il abandonné sa traduction sans que l'on sache exactement quelle est la part du travail réalisé par José María Quiroga Plá.

\footnotetext{
${ }^{2}$ À propos de l'influence de Proust sur la poésie de Salinas, on pourra lire: Ilena Antici «Du côté de chez Salinas: un poeta che legge Proust». Quaderni Proustiani. $\mathrm{N}^{\circ}$ 8. Associazione Amici di Marcel Proust. 2014: 91-102. Imprimé.
} 
Le titre choisi par le ou les traducteur(s) est discutable: El mundo de Guermantes. On perd en effet le parallélisme qui existe entre le premier et le troisième tomes, celui de Swann et celui des Guermantes, autour du terme «côté» qui revêt une importance capitale dans le déroulement et la fin de la Recherche. La traduction de ce mot "côté» dans les deux titres pose d'ailleurs toujours problème aux traducteurs de Proust, et ce, quelle que soit la langue dans laquelle ils traduisent.

Un autre problème, d'un genre totalement différent, surgit dans ce tome: dans les deux éditions (l'édition française comme l'édition espagnole) est publié avec le troisième tome (Le côté de Guermantes) le premier chapitre du tome 4, intitulé Sodome et Gomorrhe I, Sodoma y Gomorra I. Dans ce chapitre, Proust évoque ce qu'il appelle, dans une expression qui doit beaucoup à Balzac, les «races maudites», les homosexuels, «descendants» de ceux qui furent châtiés par le feu envoyé par Dieu sur les villes pécheresses évoquées au chapitre 19 de la Genèse. Ce petit détail éditorial eut des conséquences majeures: le prestige de Proust diminua aussitôt en Espagne. On peut s'en étonner, dès le premier volume, l'histoire de la fille Vinteuil, vue avec sa compagne à Montjouvain par le Narrateur (dans une scène de voyeurisme qui retrouvera des échos par la suite tout au long de la Recherche) annonçait déjà ce thème. Quoi qu'il en soit l'évocation très frontale de l'homosexualité eut pour conséquences de nombreuses réactions négatives dans la presse critique. Par la suite, la Guerre Civile en Espagne empêcha la poursuite de la publication et à la prise de pouvoir de Franco, les critiques envers les mœurs présentées par Proust dans la Recherche se changèrent en insultes contre Proust. Le contexte homophobe violemment hostile et la censure franquiste feront que la traduction de Proust signeront le coup d'arrêt de la traduction de Proust en Espagne après ce troisième tome. L'Histoire vient donc interrompre le travail de traduction et de diffusion de la grande œuvre de Proust et l'Espagne qui avait été pionnière dans ce domaine va prendre beaucoup de retard sur le monde anglophone notamment.

Ce qui ne pouvait plus se faire en Espagne s'est donc continué en Argentine puisque c'est un éditeur argentin, Santiago Rueda, qui a pris le relai. Il réédite en 1944 et 1945 les tomes déjà existants de la traduction Salinas/Quiroga Plá et leur ajoute progressivement les quatre tomes manquants en faisant appel à un nouveau traducteur, Marcelo Menasché. Ainsi, en 1946, les lecteurs hispano-américains peuvent lire la Recherche en totalité. Rueda continue son entreprise en publiant les œuvres de jeunesse, un choix de correspondance, les pastiches mais aussi des essais dédiés à l'écrivain français, et ce, en seulement quelques années. Il fait toujours appel au même traducteur qui possédait une capacité de travail assez remarquable compte tenu de la quantité de texte assez colossale qu'il a traduite en seulement quelques années.

Il faudra attendre 1952 pour que le travail commencé en Espagne reprenne, chez un autre éditeur. Le programme éditorial de Rueda s'est révélé très ambitieux et c'est par un autre programme ambitieux que va lui répondre l'éditeur barcelonais Plaza y Janés. Il consacre à Proust trois volumes de sa collection «Clásicos del siglo XX», deux pour En busca del tiempo perdido, le second débutant avec Sodoma y Gomorra, et un troi- 
sième volume regroupant divers textes de Proust (Jean Santeuil, Les Plaisirs et les Jours et quelques lettres, entre autres). Le fait que le second volume commence avec Sodoma y Gomorra fait que ce second volume a été interdit à la vente par la censure franquiste pendant des années alors que le premier volume (contenant tout de même l'épisode de Montjouvain) n'a valu aucune inquiétude à son éditeur. Là encore la différence de traitement peut surprendre de la part de la censure, l'homosexualité féminine posant vraisemblablement moins de problèmes que celle des hommes. ${ }^{3}$

En ce qui concerne la traduction proprement dite de cette nouvelle édition, elle est construite sur le mê principe que celle de Santiago Rueda: la traduction Salinas / Quiroga Plá est conservée pour les trois premiers tomes, et c'est un nouveau traducteur, Fernando Gutiérrez qui est chargé de compléter l'œuvre en traduisant les quatre tomes manquants et les textes contenus dans le dernier volume de cette collection «Clásicos del siglo XX»). Pour la première fois, le tout est précédé d'une assez longue introduction critique qui présente l'œuvre proustienne. Malgré le contexte politique peu favorable à tout discours positif sur Proust, un écrivain, José Luis Cano, propose dans le journal Ínsula une recension (Cano, 1953) qui vante les mérites de cette nouvelle traduction par rapport à la traduction argentine de Marcelo Menasché.

Une dizaine d'années plus tard, l'éditeur Alianza editorial propose sa propre édition de la Recherche, sous la forme d'une édition de poche (pour la première fois) en sept volumes entre 1966 et 1969; la censure s'est alors assouplie puisque cette édition ne verra pas d'obstacles à sa diffusion. On conserve toujours le même système puisque la traduction des trois premiers tomes est reprise telle quelle et que c'est un autre traducteur - cette fois-ci une traductrice - qui complète en traduisant les quatre derniers tomes. Il s'agit d'une traductrice d'expérience, Consuelo Berges, qui a déjà à son actif La Bruyère, Flaubert ou encore Stendhal. Cette nouvelle traduction pourrait surprendre car elle intervient peu de temps après la précédente, mais elle est en réalité tout à fait justifiée par l'actualité proustienne de l'autre côté des Pyrénées. En 1954 paraît en France une nouvelle édition $\mathrm{d}^{\prime} \grave{A}$ la recherche $d u$ temps perdu, la première édition de la prestigieuse collection de la Pléiade (Proust, 1954), dont le texte a été entièrement révisé et pour lequel de nouveaux manuscrits - ignorés dans l'édition précédente- ont été pris en compte. C'est donc une nouvelle Recherche que l'on lit désormais en France et cela a justifié une nouvelle traduction espagnole. ${ }^{4}$ Consuelo Berges ajoute en outre quelques notes à son édition, malheureusement, ces notes font état d'une lecture de la traductrice qui n'est pas toujours très juste.

\footnotetext{
${ }^{3}$ On remarquera d'ailleurs que le premier volume contenant la Recherche a fait l'objet de deux tirages alors que le second n'en a eu qu'un seul. S'il est courant dans le cas de Proust que les premiers tomes de l'œuvre se vendent beaucoup plus que les suivants (preuve sans doute que de nombreux lecteurs s'arrêtent en cours de route!), l'effet de la censure franquiste est très net.

${ }^{4}$ Le copyright de la traduction de C. Berges indique néanmoins de façon fautive comme édition de référence l'édition de la collection «Blanche» de Gallimard (1919-1927) et non celle de la Pléiade.
} 
Pour ne citer que quelques exemples, il arrive régulièrement aux personnages de Proust de commenter des toponymes en convoquant les étymologies latines. À propos de l'un de ces passages, C. Berges écrit en note «en español en el texto» (Berges, IV: 539): cela veut dire qu'elle confond l'espagnol et le latin. ${ }^{5} \mathrm{D}$ 'autres exemples ont des conséquences plus fâcheuses. Albertine se met en colère contre le Narrateur à cause de la jalousie maladive de ce dernier qui l'empêche de sortir pour retrouver ses amies, elle lui dit alors: «j'aime bien mieux que vous me laissiez une fois libre pour que j'aille me faire casser... le pot» (Proust, 1987, III: 840). Le narrateur ne comprend pas sur le coup le dernier de la phrase, ce n'est qu'après qu'il comprendra. C. Berges ne traduit jamais les expressions argotiques du texte proustien, elle les laisse en français et propose une note en bas de page pour éclairer l'expression. Dans ce cas présent, elle met en relation «se faire casser le pot» avec une autre expression de l'époque, «casser la cruche», qui comme le note Consuelo Berges, signifie bien «perdre sa virginité» (Berges, V: 369). En revanche, «se faire casser le pot» signifie en réalité se faire sodomiser, ce qui fait qu'Albertine appartient non seulement au côté de Gomorrhe, mais aussi au côté de Sodome, rendant ainsi ce personnage encore plus impossible à cerner (Morel joue un rôle équivalent chez les hommes). ${ }^{6}$ Par ailleurs, Albertine n'est plus exactement vierge et le Narrateur est bien placé pour le savoir. On constate donc que C. Berges a fait dans sa traduction et dans ses notes un nombre important de contre-sens plus ou moins malheureux, néanmoins cela n'a pas empêché cette édition de trouver son public et de rester à ce jour la plus vendue de Proust en Espagne. À ce jour, un peu plus d'un million de volumes a été vendu, en une trentaine d'années et de multiples réimpressions. Toutefois, elle présente un autre défaut majeur, celui d'être désormais datée puisque le texte français de Proust qui fait à l'heure actuelle n'est plus celui qui a servi de base pour le travail de C. Berges.

Quelques années après la fin de la dictature franquiste, en 1981, l'éditeur espagnol Aguilar commence à publier une nouvelle édition complète de la Recherche avec une traduction de Julio Gomez de la Serna. La publication s'interrompt après le second tome, Les jeunes filles, et il se murmure que le manuscrit donné par le traducteur aurait été perdu chez l'éditeur (chose assez incroyable, il faut bien le dire!) ou bien que le traducteur serait mort avant d'avoir pu terminer son travail. ${ }^{7}$ Julio Gomez de la Serna qui ne sera pas le seul traducteur à décéder avant d'avoir pu terminer son travail, en d'autres termes, Proust peut tuer...

Avec les années 2000, tout change: depuis la fin des années 1980, on dispose d'une nouvelle édition française de référence, la deuxième édition de la Pléiade (Proust, 1987), et les droits d'auteurs concernant Proust sont tombés dans le domaine public.

\footnotetext{
5 À propos d'un autre passage, elle se trompe en reliant le néologisme "charentonesque" (qui réfère à l'asile de Charenton, célèbre hôpital psychiatrique d'autrefois situé non loin de Paris) avec la Charente, région de France située sur la côte atlantique).

${ }^{6}$ Voir à ce sujet: Elisabeth Ladenson. Proust lesbien. Paris: EPEL, 2004.

${ }^{7}$ C'est en tout cas l'hypothèse que retient Herbert E. Craig (Craig, "Assessing": 191).
} 
Dès lors, trois nouvelles traductions complètes voient le jour, deux en Espagne, une en Argentine.

Carlos Manzano (également traducteur de Céline) propose chez Lumen, à Barcelone, une édition sans notes (il se contente de mettre en note la traduction des vers français laissés dans le corps du texte), publiée entre 1999 et 2009. Très rapidement, une nouvelle édition dans un format de poche apparaît et commence à connaître un certain succès.

En 2000, l'éditeur Losada publie le premier volume de la deuxième édition argentine de Proust, sans notes également, signée Estela Canto, figure des milieux lettrés porteños. Cependant, la traductrice est décédée en 1994: pour ne pas voir s'échapper des lecteurs qui auraient peur de ne pas disposer de la traduction de la Recherche dans son intégralité, l'éditeur mène une campagne publicitaire assez agressive, répétant à l'envi que la traductrice a terminé sa traduction avant de mourir. De plus, la stratégie de communication insiste sur la haute qualité de la traduction. Les tomes 2 à 5 sont publiés entre 2002 et 2005, mais il faudra attendre 2010 pour voir paraître les deux derniers. À ce moment-là, le premier tirage est déjà épuisé et les deux derniers tomes ne sont donc publiés que dans le second tirage. Même si cela démontre le succès de cette nouvelle traduction argentine, la pratique éditoriale peut surprendre. Au moment de publier le dernier tome, l'éditeur est bien obligé de reconnaître qu'il a dû faire appel à une nouvelle traductrice pour traduire Le temps retrouvé, Graciela Isnardi.

En Espagne, un autre projet, totalement différent, voit le jour au même moment. Valdemar propose une édition de la Recherche laissée aux soins de Mauro Armiño (traducteur déjà reconnu), il s'agit de la première édition critique hispanique de l'œuvre de Proust. Elle se présente sous la forme de trois imposants volumes publiés entre 2000 et 2005 avec de nombreuses notes, une introduction et divers index et dictionnaires intégrés (des personnages, des lieux...), une biographie, une bibliographie, etc. Mauro Armiño continue à traduire Proust: lettres, œuvres de jeunesse, Jean Santeuil pour Valdemar. Il est à l'heure actuelle le seul à avoir traduit la quasi-totalité de l'œuvre de Proust en espagnol à lui seul.

Ce sont donc ces six éditions qui composent le corpus de traductions de la Recherche.

\section{Le débat: Espagne vs Argentine}

L'un des aspects les plus surprenants de ce débat sur la comparaison entre les différentes traductions est le fait que lorsque les traducteurs prennent la parole, ils n'évoquent presque jamais les autres traductions que les leurs. Ils parlent de leur traduction, de la nécessité d'actualiser les traductions compte tenu des nouvelles découvertes de la génétique proustienne, etc. mais dans une sorte d'élan d'humilité, ils semblent s'interdire de parler de la qualité des traductions. Du reste, lorsqu'ils s'expriment, ils le font dans des revues, des journaux mais bien peu au sein de leur édition: 
les préfaces de traducteurs - lorsqu'il y en a— restent toujours très neutres. Par conséquent le débat sur les traductions - qui fut parfois violent — s'est fait presque sans la parole des traducteurs.

L'édition Rueda, avec la traduction de Marcelo Menasché, fut énormément critiquée dans les années 1940-1950, surtout de la part de lecteurs espagnols et parfois latino-américains. Malgré cela, elle connut un certain succès puisqu'elle se diffusa dans toute l'Amérique Latine mais peu en Espagne. Les arguments furent presque toujours les mêmes: la traduction était pleine de «modismos» argentins et se révélait très mauvaise en comparaison de celle qu'avait donnée Pedro Salinas. Jusque dans les années 1980, personne n'a critiqué celle de Salinas. Il y avait une sorte de consensus sacralisant de son travail. Certains articles critiques très virulents à l'égard de $\mathrm{M}$. Menasché élaborent un discours très vague sans jamais citer aucun exemple à l'appui. Le cas le plus remarquable à cet égard est probablement celui de José Luis Cano qui publie un article élogieux à propos de la traduction réalisé par Fernando Gutiérrez dans lequel il condamne la traduction de Menasché en deux phrases: à se demander s'il a véritablement lu de près le travail de Menasché ou si son article sert simplement à valoriser la traduction concurrente. Le doute est permis d'autant que quelques temps plus tard, J. L. Cano republie son article dans un livre chez Plaza y Janés, ${ }^{8}$ l'éditeur de la traduction de Fernando Gutiérrez. Peut-être que les intérêts personnels ont pris le dessus sur l'objectivité. Si la version de Menasché n'est pas excellente, elle n'est pas si mauvaise que cela, en tout cas, elle n'est pas si inférieure à celle de Salinas qui est loin d'être exempte de défauts et même de contresens majeurs. Le plus célèbre concerne le titre du second chapitre de Du côté de chez Swann, «Un amour de Swann» que Salinas traduit de manière assez incompréhensible par «Unos amores de Swann», ce qui est le plus gros contresens que l'on pouvait faire au vu de ce que raconte Proust dans cet épisode. ${ }^{9}$ D'ailleurs si Menasché et Rueda, de même que Gutiérrez et Plaza y Janés corrigèrent cette erreur, Consuelo Berges et Alianza Editorial la laissèrent telle quelle.

Ce qui peut le plus surprendre dans ce débat concernant la qualité des différentes traductions est probablement la mauvaise foi ou le manque de connaissances des journalistes et critiques qui traitèrent le sujet. En vérité, aucun d'entre eux ne semble bien connaitre les problèmes que pose de la traduction proustienne. Pour avoir parcouru environ une vingtaine de recensions ou d'entretiens, on est systématiquement confronté aux mêmes insuffisances.

Les critiques littéraires n'ont pas procédé à un travail de documentation sur le sujet. Certains traitent de toutes les traductions espagnoles, y compris celles qui sont incomplètes, mais en revanche, ils ne disent pas un mot sur l'existence des traductions

\footnotetext{
${ }^{8}$ Voir Cano, "Proust visto, por los españoles".

${ }^{9}$ Voir Craig, "Assessing the Spanish..." (parmi ses articles sur les traductions de Proust, c'est le plus complet et il synthétise tous les autres) et Jover Silvestre.
} 
argentines. La dernière traduction argentine, celle d'Estela Canto, n'apparaît par exemple presque jamais dans les journaux et revues espagnoles.

Un phénomène similaire se passe avec les traducteurs qui n'évoquent, eux, que des problèmes techniques, difficultés liées à la syntaxe, hésitations lexicales... Il est évidemment important qu'ils abordent le sujet d'un point de vue plus technique, mais néanmoins, lorsqu'ils font référence —en général très rapidement- aux traductions antérieures, les traducteurs espagnols semblent totalement occulter les traducteurs argentins et la réciproque est quasiment vraie.

Les lecteurs espagnols ignorent donc à peu tout de ce qui se passe de l'autre côté de l'Atlantique. Est-ce par ignorance ou par volonté de ne pas savoir à cause d'une rivalité entre les deux nations? Ou bien, est-ce parce que l'on considère que l'on n'y parle pas tout à fait la même langue? Quoiqu'il soit, la traversée de l'Atlantique n'est guère aisée...

Les articles consacrés au sujet et publiés en Argentine furent beaucoup moins nombreux qu'en Espagne. L'un d'entre eux se révèle toutefois très intéressant et fut publié dans La Nación en 2005. Il est signé d'Herbert E. Craig, spécialiste du sujet. Quelques années avant la parution de cet article, en 2001 plus précisément, il avait écrit: "A pesar de lo antedicho, no encuentro mucha diferencia de nivel entre la versión de Canto y las otras nuevas. En su selección de palabras y expresiones, Canto refleja hasta cierto punto su dialecto sudamericano y argentino, pero su lenguaje nunca deja de ser pulido ni cae en lo meramente coloquial. Asimismo evita el tipo de argentinismo que se le achacaba a la versión de Menasché de los últimos tomos de Proust" (Craig, "Marcel Proust de nuevo": 140).

Craig explique que les trois dernières traductions lui semblent de qualité similaire. Le propos, plutôt prudent, se retrouve dans l'article de 2005:

Es quizás en Sodoma y Gomorra (el tomo proustiano que otro argentino dio por primera vez al mundo hispánico y en el cual ni Manzano se muestra tan brillante ni Armiño tan erudito) donde es posible ver mejor el modo en que Canto sí sabía defenderse como traductora de Proust. Ella presenta, con el mismo nivel de capacidad que los dos nuevos traductores españoles y en un grado más alto que los anteriores de ambos lados del Atlántico, ese texto muy original y polémico del novelista francés.

Le chapeau introductif à cet article de 2005 adopte un ton un peu différente et peut laisser penser qu'il n'est pas de Craig: "Recientemente las traducciones al español de En busca del tiempo perdido se han multiplicado. El autor de Marcel Proust and Spanish America, especialista en el tema, considera los valores y las diferencias de las más conocidas, entre las que sobresale la realizada por Estela Canto".

L'emploi du verbe sobresale a en effet de quoi surprendre: à aucun moment Craig ne dit que la traduction d'Estela Canto surpasse les autres traductions. Le journaliste (qui a signé le chapeau introductif) paraît donc se révéler plus enthousiaste vis-à-vis de sa compatriote que ne l'est le spécialiste. 
L'éditeur d'Estela Canto, Losada, mentionne en quatrième de couverture la qualité de sa traduction (ce que ne Font pas les autres éditeurs): "Con la excelente traducción de la escritora argentina Estela Canto, la obra de Marcel Proust ingresa por primera vez a una lengua familiar, cercana, distinta de las traducciones españolas que incluyen giros y modismos en ocasiones tan perturbadoras por la lectura".

Losada procède donc à une double opération de valorisation de sa propre traduction et de dénigrement des traductions concurrentes espagnoles. C'est le même mot de modismos qui est utilisé et que l'on rencontrait déjà dans les articles (majoritairement espagnols) très critiques sur la traduction de Marcelo Menasché. Le paradigme lexical fait donc un aller-retour entre les deux rives de l'Atlantique.

Lors de la réédition de la traduction d'E. Canto chez Losada, cette quatrième de couverture assez agressive a disparu (comme Albertine, pourrions-nous dire). Était-ce parce qu'elle était trop agressive ou bien parce qu'elle mentionnait malgré tout l'existence d'autres traductions qu'il valait mieux cacher? On constate donc que depuis 70 ans, les mots, les formules et la rhétorique n'ont guère évolués.

\section{Bilan provisoire}

Dans leur majorité, les lecteurs espagnols lisent un Proust espagnol et les hispano-américains un Proust argentin; tous les hispanophones ne lisent donc pas le même Proust, ils lisent la même œuvre, mais pas le même texte. La question de la traduction est ainsi parasitée par des problèmes externes, politico-culturels et commerciaux.

La confrontation n'est pas encore terminée: l'Espagne a gagné une première manche, par 4 traductions contre 2. Mais la situation va probablement évoluer rapidement. En effet, les règles de diffusion de œuvres ont significativement changé: l'arrivée d'internet et de la vente en ligne ont largement assoupli les communications entre Espagne et Amérique Latine et les pratiques éditoriales. Deux des six traductions sont aujourd'hui épuisées (celle de Menasché et celle de Gutiérrez), et il semble peu probable qu'un autre éditeur s'aventure à vouloir publier une nouvelle traduction car le marché paraît un peu saturé. Néanmoins, si cela arrive, on peut penser qu'il s'agira d'une traduction mexicaine par exemple ou cubaine, en tout cas utilisant un espagnol différent de celui usité en Espagne ou en Argentine.

Le lecteur peut donc choisir aujourd'hui entre quatre traductions. Celle de Consuelo Berges pose problème dans la mesure où elle s'appuie sur un texte dépassé et fautif et que ses notes sont largement critiquables. Cela étant, les Espagnols sont encore attachés à cette traduction, elle demeure la traduction traditionnelle de Proust. Le futur de cette traduction dépendra du comportement d'Alianza Editorial: si l'éditeur n'accepte pas de réviser le texte, elle va perdre rapidement de son intérêt et risque d'être délaissée par les lecteurs. 


\section{Comparaison}

Ce type d'étude possède un passage obligé, celui de la comparaison des qualités respectives de chaque édition pour essayer de déterminer quelle est la meilleure. Je me trompe peut-être, mais à terme, on peut penser que celle de Consuelo Berges va disparaître. Elle sera probablement remplacée, peu à peu, par celle de Carlos Manzano, l'autre version de poche. Aujourd'hui, il n'est plus possible de lire la version dépassée de C. Berges, il reste donc les trois éditions des années 2000. Il faut d'abord comparer les éditions avant de comparer les traductions.

- Carlos Manzano: son édition est une édition de poche en 7 volumes, publiée au départ par Debolsillo, aujourd'hui chez RBA. Elle ne comporte à peu près aucune note, son prix est plus modéré. Le traducteur traduit tout, y compris les citations, dans le corps du texte.

- Celle d'Estela Canto, chez Losada, est également publiée en 7 volumes un peu plus grands, plus chers aussi et ne comporte pas de notes. Elle traduit tout y compris les citations.

-L'édition proposée par Mauro Armiño chez Valdemar est radicalement différente. Vendue dans un coffret sous la forme de trois énormes volumes, elle est très difficilement transportable. C'est une édition critique assez remarquable, de type universitaire presque, très complète qui comprend de nombreuses notes, différents index (personnages, lieux...), une introduction générale, une biographie et une bibliographie. Armiño conserve les citations en français dans le texte et en propose une traduction en note. Son prix est à peu près le doublé de celle de Manzano.

Cette dernière est évidemment de loin la meilleure édition; si l'on doit faire un travail sur le texte de Proust sans pouvoir recourir au texte français, c'est cette édition qui s'impose. Pourtant, le prix de celle de Manzano devrait lui assurer un succès populaire.

En ce qui concerne les traductions proprement dites, la traduction de Manzano est celle qui se démarque le plus des autres (y compris en tenant compte des anciennes traductions). Les traductions de Canto et d'Armiño sont plus proches l'une de l'autre.

La traduction de Manzano est vive, généralement assez concise et elle utilise une langue plutôt moderne et transpose les culturèmes; mais à mon avis, elle souffre d'un problème majeur, elle ne respecte pas du tout la ponctuation de Proust qui est si particulière et fait partie du style de l'écrivain. En effet Manzano coupe les phrases (y compris les très longues si caractéristiques), les restructure et ainsi perd le rythme propre de la phrase proustienne. Sa traduction est clairement cibliste. La traduction d'E. Canto est une bonne traduction, mais sa tendance à peut-être un peu moderniser la langue de Proust (au niveau du lexique par exemple) fait parfois perdre la saveur «années folles» ou «fin de siècle» du roman proustien. La traduction d'Armiño est incontestablement sérieuse mais peut par moments, manquer de vivacité, elle paraît un peu plus académique en quelque sorte. C'est lui qui néanmoins suit le plus le texte 
original, sa traduction est plutôt sourcière. Canto et Armiño conservent plus ou moins les culturèmes, Canto me semble un peu moins sourcière qu'Armiño, mais sans être cibliste pour autant.

Les trois traductions, malgré quelques contresens ou erreurs dus à des fautes d'inattention (il est bien difficile de ne pas en commettre dans une traduction de 3000 pages), sont de bonnes traductions. Chacune a son originalité, sa tonalité propre.

À titre de comparaison, nous avons isolé deux phrases tirées du premier tome, $D u$ côté de chez Swann, Por el lado de Swann.

Quelquefois, comme Ève naquit d'une côte d'Adam, une femme naissait pendant mon sommeil d'une fausse position de ma cuisse. Formée du plaisir que j'étais sur le point de goûter, je m'imaginais que c'était elle qui me l'offrait (Proust, 1987, I: 4).

Carlos Manzano propose la traduction suivante:

A veces, durante mi sueño, una mujer nacía — como Eva de una costilla de Adán— de una mala postura de mi muslo. Me imaginaba que era ella — creada por el placer que estaba a punto de experimentar — quien me lo ofrecía (Manzano, I: 11).

On voit d'emblée les tirets utilisés par Manzano qui n'appartiennent pas au texte original. De plus, Proust n'utilise que rarement les tirets qui par ailleurs, surtout à son époque, ne sont pas un signe de ponctuation très usité en français. Ces tirets facilitent sans doute la lecture pour le lecteur espagnol mais peut sembler étrange à un lecteur qui connaît Proust parce que cela ne lui ressemble pas. Le travail de reconstruction mentale de la structure de la phrase lorsqu' on lit Proust fait partie de la lecture de Proust. C'est un peu dommage de perdre cet aspect ici. Cet usage du tiret est un élément significatif du caractère cibliste de la traduction de Manzano.

Estela Canto adopte une ponctuation beaucoup plus proche de celle de Proust:

Algunas veces, así como Eva había nacido de una costilla de Adán, una mujer nacía durante mi sueño de una falsa posición del muslo. Formada por el placer que estaba a punto de disfrutar, yo me imaginaba que era ella quien me lo ofrecía (Canto, I: 15).

Les variantes qu'elle offre avec celle de Mauro Armiño dans ce passage sont minimes:

Algunas veces, lo mismo que Eva nació de una costilla de Adán, una mujer nacía durante mi sueño de una falsa postura de mi muslo. Nacida del placer que yo estaba a punto de gozar, imaginaba que era ella quien me lo ofrecía (Armiño, 2000, I: 8).

Les trois traductions dans l'ensemble sont assez proches les unes des autres (si on laisse de côté la ponctuation) et la différence est surtout marquée vis-à-vis des 
anciennes traductions. On peut aisément partager l'opinion d'Herbert E. Craig: elles sont de qualité plus ou moins équivalente. L'édition Losada pâtit néanmoins du fait que ce sont deux traductrices et non une seule qui ont accompli le travail. Évidemment Graciela Isnardi, la seconde traductrice, a suivi les choix d'Estela Canto lorsqu'elle a dû terminer le travail de celle-ci mais ici ou là, on relève quelques incohérences très ponctuelles. Par exemple, pour renvoyer à la pâtisserie française désignée sous le terme de «croissant», Estela Canto utilise le terme medialuna (Canto VI: 101) alors que sa suivante opte le mot français, en italiques, "croissant", avec son orthographe originale (Canto VII: 100). ${ }^{10}$ Elle est en somme ici plus sourcière que celle qui l'a précédée. Cette édition, comme celle de Manzano, souffre aussi du manque de notes et il est assez évident que certaines allusions de Proust exigent un éclaircissement pour $90 \%$ des lecteurs d'aujourd'hui, qui plus est, lorsqu'il s'agit de lecteurs hispanophones. C'est pourquoi l'édition d'Armiño se détache un peu des autres, même si, malgré son admirable travail, il manque quelques notes chez lui aussi! ${ }^{11}$

En guise de conclusión, il convient de souligner que les traductions de Proust en espagnol offrent un corpus des plus remarquables pour les études de traductologie, avec une trajectoire assez singulière. Chaque traduction a son importance dans l'histoire de la diffusion de l'œuvre de Proust dans le monde hispanique, qu'elle soit la première, qu'elle réactualise le texte, ajoute des notes ou une introduction critique... Les traductions des années 2000 sont particulièrement remarquables, de grande qualité, et elles permettent au lecteur de choisir celle qui lui convient le plus, ce qui est peu fréquent pour un auteur du $\mathrm{XX}^{\mathrm{e}}$ siècle. ${ }^{12}$

\section{Bibliographie}

\section{Éditions françaises de Marcel Proust}

Proust, Marcel. À la recherche du temps perdu. 2a édition de "la Pléiade", sous la direction de Jean-Yves TADIÉ. 4 volumes. Paris: Gallimard, 1987. Imprimé.

${ }^{10}$ Armiño utilise le mot français mais en l’hispanisant en "cruasán” (Armiño, III: 668).

${ }^{11}$ Par exemple, dans le dialogue qui suit, Mme de Guermantes fait un jeu de mots sur le nom de son neveu, Robert de Saint-Loup, jeu de mots incompréhensible au lecteur ne connaissant pas le français si on ne le lui explique pas:

“-Vaya, en hablando del Saint-Loup..., dijo Mme de Guermantes.

$[\ldots]$

— ¡Ah!, en hablando del Saint-Loup, ahora comprendo, dijo el diplomático belga riendo a carcajadas.

-Delicioso — replicó secamente Mme de Guermantes, que detestaba los juegos de palabras y sólo había aventurado aquél fingiendo burlarse de sí misma” (Armiño, II: 227).

En français, il existe un proverbe qui dit "Quand on parle du loup, on en voit la queue" (qui équivaut au proverbe espagnol, "Hablando del rey de Roma...").

${ }^{12}$ Une version un peu différente de cette étude a déjà été publiée en France. 
Contre Sainte-Beuve. Édition de Pierre Clarac e Yves SANDre. Paris: Gallimard, 1971. Imprimé.

Jean Santeuil, précédé de Les plaisirs et les jours. Édition de Pierre ClARAC et Yves SANDRE. Paris: Gallimard, 1971. Imprimé.

À la recherche du temps perdu. $1^{\mathrm{e}}$ édition de "la Pléiade" de Pierre ClaraC et André FERRÉ. 3 volumes. Paris: Gallimard, 1954. Imprimé.

À la recherche du temps perdu. 15 volumes. Paris: Gallimard / NRF, 1919. Imprimé.

Éditions de En busca del tiempo perdido en espagnol

Proust, Marcel. En busca del tiempo perdido. Trad. Carlos MANZANo. Barcelona: Lumen, 2006-2010. 7 volumes. Imprimé.

A la busca del tiempo perdido. Trad. Mauro ArMIÑo. Madrid: Valdemar, 2007. 3 volumes. Imprimé.

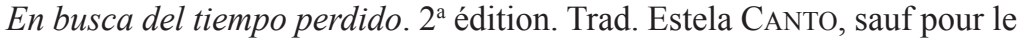
volume 7, El tiempo recuperado, trad. Graciela IsNARDI. Buenos Aires: Losada, 2004-2010. 7 volumes. Imprimé.

. En busca del tiempo perdido. $1^{a}$ édition (incomplète). Trad. Estela CANTO. Buenos Aires: Losada, 2000-2005. 5 volumes. Imprimé.

. En busca del tiempo perdido. Trad. Pedro Salinas, José María Quiroga Pla et Marcelo Menasché. Buenos Aires: Santiago Rueda, 1979-1980. 7 volumes. Imprimé.

. En busca del tiempo perdido. Trad. Pedro Salinas, José María Quiroga Pla et Fernando GuTIÉRREZ. Barcelona: Plaza y Janés, 1967-1968. 2 volumes. Imprimé.

En busca del tiempo perdido. $1^{\mathrm{e}}$ édition. Trad. Pedro Salinas, José María Quiroga Pla et Consuelo Berges. Madrid: Alianza Editorial, 1966. 7 volumes. Imprimé.

\section{Bibliographie critique et journalistique}

ArmiÑo, Mauro. "Proust, piedra de toque". El Siglo. Web. 03 sep. 2007. $<$ http:// www.elsiglodeuropa.es/siglo/historico/2007/752/752pens.html>.

BERges, Consuelo. "La traducción y mi traducción de Proust". El Urogallo. 1971: 71-76. Imprimé.

Blanco, José Joaquín. "Proust proliferado". La iguana del ojete —Blog de José Joaquin Blanco. Web. 07 jul. 2010. <http://iguanadelojete.blogspot.com/ 2008/10/proust-proliferado.html>. 
CANO, José Luis. "Proust, visto por españoles". El escritor y su aventura. Barcelona: Plaza y Janés, 1966. Imprimé.

"Proust en castellano". Ínsula. 15 ago. 1953: 6-7. Imprimé.

CONTE, Rafael. "A cada cual su Proust". Babelia, suplemento cultural de El País. Web. 20 sep. 2001. <http://www.elpais.com/articulo/narrativa/Proust/ elpbabnar/20010929elpbabnar_7/Tes>.

CRAIG, Herbert. "Proust en España durante los tiempos de Franco". Marcel Proust: écriture, réécritures / Marcel Proust: escritura, rescrituras. Bruxelles: Peter Lang, 2010. 355-365. Imprimé.

"Sodoma y Gomorra, el tomo más polémico y traducido de Proust". Chasqui. 2005: 149-153. Imprimé.

. "Sobre los traductores de Proust". Lanación.com. Web. Nov., 2005. $<$ http://www.lanacion.com.ar/753523-sobre-los-traductores-de-proust $>$. . Marcel Proust and Spanish America (from critical response to narrative dialogue). Lewisburg: Bucknell University Press, 2002. Imprimé.

. "Pedro Salinas as Proust's First Translator". Confluencia. 2002. 129-138. Imprimé.

"Assessing the Spanish translations of Proust". Proceedings of the $42^{\text {nd }}$

Conference of the American Translators. 2001. 191-201. Imprimé.

"Marcel Proust de nuevo en español". Chasqui. Mayo, 2001. 137-141. Imprimé.

"Proust en España y en Hispanoamérica". Bulletin hispanique. 1999. 75185. Imprimé.

Jover Silvestre, Yolanda. "Proust: traducción y recepciones". La traducción: puente interdisciplinar. Almería: Universidad de Almería, 2001. 129-151. Imprimé.

MARTER, Inka. "Recepción argentina de la obra de Marcel Proust". Recuerdo y voz. La narrativa de Norah Lange en sus contextos. 122-136. Web. 2011. <kups. ub.uni-koeln.de/3160/1/Marter___Recuerdo_y_voz.pdf>.

MiRANDA, Virginia. “'Proust alteró el concepto de novela'. Entrevista con Mauro Armiño". El Siglo. Web. 20 jun. 2005. <http://www.elsiglodeuropa.es/siglo/ historico/Pensamiento/pens2005/654pens.htm>.

Montesinos, Toni. "Última lectura de Proust", LaRazón.es. Web. 21 mayo 2009. $<$ http://www.larazon.es/noticia/ultima-lectura-de-proust>.

RODRÍGUEZ, Emma. "Nuevo viaje a las entrañas de Proust". Elmundolibro.com. Web 12 jun. 2000. <http://www.elmundo.es/elmundolibro/2000/06/12/anticua rio/960809091.html>.

Rodríguez MARCos, Javier. "Homero no escribía en español". Babelia, suplemento cultural de El País, Web 28 oct. 2006. <http://www.acett.org/documentos /20061028babelia.pdf>.

Ruiz de Huydobro, Roberto. "Tiempo para Proust". Pérgola, suplemento del periódico Bilbao. Web. Nov., 2005. 4. <http://www.bilbao.net/cs/Satellite?c=BIO_ 
Publicacion_FP\&cid $=3000063004 \&$ language $=$ es\&pageid $=3000018331 \&$ pag ename $=$ Bilbaonet\%2FBIO_Publicacion_FP\%2FBIO_GrupoPublicacion> .

SAlADRIGAS, Robert. "Los riesgos de traducir a Proust". Revista de libros. Web. Mar., 2001. $<$ http://www.revistadelibros.com/articulo_completo.php?art $=3888>$.

VILLENA, Luis Antonio de. "A la busca del tiempo perdido (I)". Elcultural.es. Web. 12 jul. 2000. $<$ http://www.elcultural.es/version_papel/LETRAS/1905/A_la_ busca_del_tiempo_perdido_(I)/>. 
